\title{
Erratum: Topological bulk lasing modes using an imaginary gauge field [Phys. Rev. Research 3, 033042 (2021)]
}

\author{
Stephan Wong (1) and Sang Soon Oh
}

(Received 13 January 2022; published 31 January 2022)

DOI: 10.1103/PhysRevResearch.4.019001

We have noted some errors in this paper. These are only typographical errors in equations and do not affect the results obtained.

Equation (18) in our original paper should read

$$
\tilde{H}_{I \leftarrow J}^{\dagger} \psi_{I, 0}^{(i)}+r_{i} \tilde{H}_{J \rightarrow I}^{\dagger} \psi_{I, 0}^{(i)}=0 .
$$

In addition, the sizes and the explicit expressions of the coupling matrices were not correctly typed in. The sizes of the coupling matrices $H_{I \leftarrow J}$ and $H_{J \rightarrow I}$ should be $\left(N_{s} \times N_{s}^{\prime}\right)$ where $N_{s}$ and $N_{s}^{\prime}$ are the number of sites in the $I$ and $J$ lattices, respectively.

For the two-dimensional (2D) kagome lattice, the Hermitian coupling matrices should be written as

$$
\tilde{H}_{I \leftarrow J}^{\dagger}=H_{I \leftarrow J}^{\dagger}=\left(\begin{array}{cccccc}
t_{1} & t_{1} & 0 & \cdots & \cdots & \cdots \\
\cdots & 0 & t_{1} & t_{1} & 0 & \cdots \\
\cdots & \cdots & \cdots & \cdots & \cdots & \cdots \\
\cdots & \cdots & \cdots & \cdots & 0 & t_{1}
\end{array}\right),
$$

and

$$
\tilde{H}_{J \rightarrow I}^{\dagger}=H_{J \rightarrow I}^{\dagger}=\left(\begin{array}{cccccc}
t_{2} & 0 & \ldots & \ldots & \cdots & \cdots \\
0 & t_{2} & t_{2} & 0 & \cdots & \cdots \\
\ldots & \cdots & \ldots & \ldots & \ldots & \ldots \\
\ldots & \ldots & \ldots & 0 & t_{2} & t_{2}
\end{array}\right),
$$

whereas, for the non-Hermitian case, they should read

$$
\tilde{H}_{I \leftarrow J}^{\dagger}=\left(\begin{array}{cccccc}
t_{1} e^{h^{\prime}} & t_{1} e^{h^{\prime \prime}} & 0 & \ldots & \cdots & \cdots \\
\cdots & 0 & t_{1} e^{h^{\prime}} & t_{1} e^{h^{\prime \prime}} & 0 & \cdots \\
\cdots & \cdots & \cdots & \cdots & \ldots & \ldots \\
\cdots & \cdots & \ldots & \ldots & 0 & t_{1} e^{h^{\prime}}
\end{array}\right),
$$

and

$$
\tilde{H}_{J \rightarrow I}^{\dagger}=\left(\begin{array}{cccccc}
t_{2} e^{-h^{\prime}} & 0 & \cdots & \cdots & \cdots & \cdots \\
0 & t_{2} e^{-h^{\prime \prime}} & t_{2} e^{-h^{\prime}} & 0 & \cdots & \cdots \\
\cdots & \cdots & \cdots & \cdots & \cdots & \cdots \\
\cdots & \cdots & \cdots & 0 & t_{2} e^{-h^{\prime \prime}} & t_{2} e^{-h^{\prime}}
\end{array}\right) .
$$

For the Lieb lattice described in the Appendix, Sec. 1, the Hermitian coupling matrices should be as follows:

$$
\tilde{H}_{I \leftarrow J}^{\dagger}=H_{I \leftarrow J}^{\dagger}=\left(\begin{array}{cccccc}
t_{3} & 0 & \ldots & \ldots & \ldots & \cdots \\
\cdots & 0 & t_{3} & 0 & \ldots & \ldots \\
\cdots & \ldots & \ldots & \ldots & \ldots & \ldots \\
\cdots & \ldots & \ldots & \ldots & 0 & t_{3}
\end{array}\right),
$$

and

$$
\tilde{H}_{J \rightarrow I}^{\dagger}=H_{J \rightarrow I}^{\dagger}=\left(\begin{array}{cccccc}
t_{4} & 0 & \ldots & \ldots & \ldots & \cdots \\
\cdots & 0 & t_{4} & 0 & \ldots & \ldots \\
\cdots & \ldots & \ldots & \ldots & \ldots & \ldots \\
\cdots & \ldots & \ldots & \ldots & 0 & t_{4}
\end{array}\right),
$$

Published by the American Physical Society under the terms of the Creative Commons Attribution 4.0 International license. Further distribution of this work must maintain attribution to the author(s) and the published article's title, journal citation, and DOI. 
whereas the non-Hermitian ones should be as follows:

$$
\tilde{H}_{I \leftarrow J}^{\dagger}=\left(\begin{array}{cccccc}
t_{3} e^{h_{2}} & 0 & \ldots & \ldots & \ldots & \ldots \\
\cdots & 0 & t_{3} e^{h_{2}} & 0 & \ldots & \ldots \\
\ldots & \ldots & \ldots & \ldots & \ldots & \ldots \\
\cdots & \ldots & \ldots & \ldots & 0 & t_{3} e^{h_{2}}
\end{array}\right),
$$

and

$$
\tilde{H}_{J \rightarrow I}^{\dagger}=\left(\begin{array}{cccccc}
t_{4} e^{-h_{2}} & 0 & \ldots & \ldots & \ldots & \cdots \\
\cdots & 0 & t_{4} e^{-h_{2}} & 0 & \ldots & \ldots \\
\cdots & \ldots & \ldots & \ldots & \ldots & \ldots \\
\cdots & \cdots & \ldots & \ldots & 0 & t_{4} e^{-h_{2}}
\end{array}\right) .
$$

For the 2D Su-Schrieffer-Heeger lattice described in the Appendix, Sec. 2, the Hermitian coupling matrices in Eqs. (A6) and (A7) should be as follows:

$$
\tilde{H}_{I \leftarrow J}^{\dagger}=H_{I \leftarrow J}^{\dagger}=\operatorname{diag}\left(t_{1}, t_{1}, \ldots, t_{1}, t_{1}\right)
$$

and

$$
\tilde{H}_{J \rightarrow I}^{\dagger}=H_{J \rightarrow I}^{\dagger}=\operatorname{diag}\left(t_{2}, t_{2}, \ldots, t_{2}, t_{2}\right),
$$

whereas their non-Hermitian counterpart in Eqs. (A11) and (A12) should read

$$
\tilde{H}_{I \leftarrow J}^{\dagger}=\operatorname{diag}\left(t_{1} e^{h_{2}}, t_{1} e^{h_{4}}, \ldots, t_{1} e^{h_{2}}, t_{1} e^{h_{4}}\right),
$$

and

$$
\tilde{H}_{J \rightarrow I}^{\dagger}=\operatorname{diag}\left(t_{2} e^{-h_{2}}, t_{2} e^{-h_{4}}, \ldots, t_{2} e^{-h_{2}}, t_{2} e^{-h_{4}}\right) .
$$

